\title{
Can't Breath: a case of unilateral diaphragmatic paralysis post CABG
}

\author{
Mohsin Khan D.O. ${ }^{*}$, Mitchell S. Karl', Ashwin Durairaj', and Antonello Santini' \\ ${ }^{* 1}$ Florida Atlantic University, Schmidt School of Medicine, 777 Glades Road, Boca Raton, FL 33431, United States \\ ${ }^{2}$ Department of Pharmacy, School of Medicine, University of Napoli Federico II, Via D. Montesano 49, 80131 Napoli, Italy
}

Article Info

\section{Article Notes}

Received: November 28, 2017

Accepted: February 01, 2018

\section{*Correspondence:}

Mohsin Khan D.O., Florida Atlantic University, Schmidt School of Medicine, 777 Glades Road, Boca Raton, FL 33431, United States; Tel.:/Fax: 0013052069648 ;

E-mail: khanm@health.fau.edu

(c) 2018 Khan M. This article is distributed under the terms of the Creative Commons Attribution 4.0 International License.

\section{Background}

Diaphragmatic paralysis is a rare cause of dyspnea one should always consider in patients of all ages. Its presentation can range in severity from being asymptomatic to acute respiratory failure ${ }^{1 \text {, }}$ ${ }^{7}$. There are a variety of treatments available to improve symptoms. However, time is the key determinant for recovery. Its etiologies can range from trauma secondary to cardiothoracic surgery, infectious diseases, malignancy, inflammatory and neuromuscular conditions $2,3,7$. In our case report, we present a 71 years old Caucasian male with an atypical presentation who was found to have unilateral diaphragmatic paralysis one-month post coronary artery bypass graft (CABG).

\section{Case Report}

A 71 year old Caucasian male with a past medical history of coronary artery disease with recent CABG x 2 vessels one month prior, presented with progressive dyspnea for 4 days. The patient stated he was able to walk a block with minimal shortness of breath post procedure, however in the past few days he developed worsening dyspnea exacerbated by talking. Patient also complained of orthopnea requiring accessory muscle usage but no chest pain or leg swelling. Patient denied a cough, fevers, chills, pleurisy, abdominal pain, nausea or vomiting. Alleviating factors included rest and exacerbating factors were any type of minimal exertions. The patient denied recent travel or medication changes.

On physical exam, the patient's vitals were: Temperature 98.8F, BP $138 / 75 \mathrm{mmHg}$, heart Rate 88 beats per minute, respiratory rate 18, Oxygen saturation $95 \%$ on room air. General: Caucasian male in mild respiratory distress. Head/Eyes/Ears/Nose/Throat: Normocephalic, atraumatic, pupils equal round reactive to light and accommodation, extraocular movements intact, jugular venous distention $4 \mathrm{~cm}$. Heart: Regular Rate Rhythm, first and second heart sounds present, no murmurs or gallops appreciated, Lungs: Decreased breath sounds in the left base, no wheezing or crackles, no dullness to percussion on bilateral lung fields. In the supine position, abdominal accessory muscles contracting for respiration. Abdomen: bowel sounds present, rebound negative, no protrusions or masses. Extremities: no pitting edema, pedal pulses 2+ bilaterally. Neuro: Awake alert and oriented to time, place, and person, no focal deficits, sensation intact bilaterally, Cranial nerves 2-12 grossly intact. His electrocardiogram reveals sinus rhythm with premature 
atrial contractions, nonspecific ST-segment changes. His initial workup with a complete blood count, basic metabolic panel, thyroid stimulating hormone level, cardiac markers and pro-BNP were unremarkable. His chest imaging displayed left basilar atelectasis. An arterial blood gas performed supine and sitting was within normal limits, confirming that the patient was not hypoxic. The patient underwent fluoroscopy with sniff test which was positive for left-sided diaphragmatic paralysis. The patient had pulmonary function tests with spirometry in the supine and sitting position which confirmed the diagnosis. The patient was started on therapy with noninvasive positive pressure ventilation (NIPPV) at nighttime and his symptomatology drastically improved. Patient was able to ambulate around the hospital floor without the need for oxygen. Patient was discharged and advised to use continuous positive airway pressure (CPAP) every night until reassessment in four weeks.

\section{Discussion}

Diaphragmatic paralysis although less common now due to advancing cardiac surgical techniques, should always be in the differential for dyspnea post cardiac surgeries regardless of a later presentation ${ }^{1}$. Unilateral diaphragmatic paralysis can present with a wide range of severity which can be managed conservatively with noninvasive positive pressure ventilation (NIPPV) all the way to surgical interventions involving plication ${ }^{3,4,7,10}$. Surgical plication is a surgical approach where folds are created in the diaphragm and these folds are then sutured in place to reduce the mobility of the paralyzed diaphragm $^{4,5}$. Our patient, although not hypoxic, received a trial of NIPPV and had positive results. Noninvasive ventilation reduces the work of breathing, improves gas exchange, relieves dyspnea and rests the inspiratory muscles $^{6,8}$. He will require daily treatment, but time will be the key component to his recovery. Usually unilateral diaphragmatic paralysis is an asymptomatic disease process unless there is a superimposed pulmonic process which hinders lung function or ventilator demands are not enough to keep up with level of physical activity ${ }^{3,4}$. We believe the patient had a delayed presentation of unilateral diaphragmatic paralysis due to his limited activity post procedure for the first few weeks causing him to mask his symptoms. The patient was advised to limit strenuous activity in the first few weeks post surgery. As the patient's level of activity increased, his symptoms started to become more apparent and he became short of breath. The patient will be followed up as an outpatient for repeat pulmonary function tests to assess for improvement. Given his delayed presentation, he will need a complete evaluation to rule out other etiologies such as neuromuscular and inflammatory disorders ${ }^{2,9}$. The patient will also need imaging of the chest to look for etiologies of nerve compression ${ }^{5}$. The time frame for recovery of unilateral diaphragmatic paralysis is case dependent ${ }^{7}$. It may require months to years to fully heal if at all ${ }^{10}$. However, with conservative management, the patient's symptoms can be lessened, in turn improving the patient's quality of life ${ }^{8}$. This finding can prompt further investigation into the conservative approach of the management for unilateral diaphragmatic paralysis. This case report also displays the importance of patient follow up and education post cardiothoracic procedures to monitor for diaphragmatic paralysis given its various presentations and how time to symptoms can be days to even weeks after procedure.

\section{References}

1. Canbaz S, Turgut N, Halici U, et al. Electrophysiological evaluation of phrenic nerve injury during cardiac surgery--a prospective, controlled, clinical study. BMC Surg. 2004; 4: 2. https://doi.org/10.1186/14712482-4-2

2. Chen, Hsuan-Yu, Chen HC, et al. Bilateral Diaphragmatic Paralysis in a Patient With Critical Illness Polyneuropathy: A Case Report. Ed. Bellou. Abdelouahab. Medicine. 94(31), 2015: e1288. PMC. Web. 27 Dec. 2017. doi: 10.1097/MD.0000000000001288.

3. Gibson GJ. Diaphragmatic paresis: pathophysiology, clinical features, and investigation. Thorax. 1989; 44: 960-970.

4. Graham DR, Kaplan D, Evans CC, et al. Diaphragmatic plication for unilateral diaphragmatic paralysis: a 10-year experience. Ann Thorac Surg. 1990; 49: 248-251.

5. Reid H, Brown J. Phrenic Nerve Decompression for the Management of Unilateral Diaphragmatic Paralysis - Preoperative Evaluation and Operative Technique. Surgical Neurology International. 2017; 8: 254. PMC. Web. 27 Dec. 2017. doi: 10.4103/sni.sni_205_17.

6. Kleopa KA, Sherman M, Neal B, et al. Bipap improves survival and rate of pulmonary function decline in patients with ALS. J Neurol Sci. 1999; 164: 82-88.

7. Kokatnur L, Rudrappa M. Diaphragm, Disorders. [Updated 2017 Oct 31]. In: StatPearls [Internet]. Treasure Island (FL): StatPearls Publishing; 2017 Jun. Available from: https://www.ncbi.nlm.nih.gov/ books/NBK470172/

8. Koopman, M, Lowie EG, Vanfeteren W, et al. Increased Exercise Tolerance Using Daytime Mouthpiece Ventilation for Patients with Diaphragm Paralysis. Breathe. 2017; 13 (3): 225-229. DOI: $10.1183 / 20734735.005817$

9. Guimarães MJ, Winck JC, B Conde B, et al. Prevalence of late-onset pompe disease in Portuguese patients with diaphragmatic paralysis DIPPER study. Revista Portuguesa de Pneumologia (English Edition). 2017; 234: 208-215.

10. Versteegh MI, Braun J, Voigt PG, et al. Diaphragm plication in adult patients with diaphragm paralysis leads to long-term improvement of pulmonary function and level of dyspnea. Eur J Cardiothorac Surg. 2007; 32: 449-456. 